\title{
Beta Chemokine
}

National Cancer Institute

\section{Source}

National Cancer Institute. Beta Chemokine. NCI Thesaurus. Code C20476.

Encoded by CC Cytokine Genes, Beta Chemokines are CC-type dual-cysteine chemokines with adjacent cysteine residues in their primary sequence that act as chemoattractants for lymphocytes, monocytes, eosinophils, and basophils, but not for neutrophils. (NCI) 